\title{
COXETER GROUPS AND QUIVER REPRESENTATIONS
}

\author{
HUGH THOMAS
}

In this expository note, I will attempt to showcase the relevance of Coxeter groups to quiver representations. The subjects which I will discuss are:

(1) real and imaginary roots,

(2) reflection functors,

(3) torsion free classes and $c$-sortable elements.

The first two of these topics are classical, going back respectively to Kac [Kac] and Bernstein-Gelfand-Ponomarev $\mathrm{BGP}$. Let $Q$ be a quiver with $n$ vertices having no oriented cycles. Associated to $Q$ is a Coxeter group $W$ which acts on $\mathbb{Z}^{n}$. Real and imaginary roots in $\mathbb{Z}_{\geq 0}^{n} \subseteq \mathbb{Z}^{n}$ are defined using $W$, and characterize the dimension vectors of the indecomposable representations of $Q$. This $W$-action on a set containing the dimension vectors of representations of $Q$ suggests the idea that $W$ might act directly on representations. Such an action does not exist. Reflection functors are, in a sense, the best we can do towards defining such an action. For this classical material I will not give proofs, but I will provide pointers to the textbooks [DDPW] and [Kir. These are particularly convenient for us since they take quiver representations as their starting point.

The third topic which I will cover is a more recent development. Torsion free classes of $\operatorname{rep} Q$ are full subcategories closed under extensions and subrepresentations. It is natural to want to classify them. It turns out that the torsion free classes that contain only finitely many isomorphism classes of indecomposables correspond to $c$-sortable elements of $W$, a notion which was introduced by Reading $\mathrm{Re}$. This correspondence was first established in Dynkin type in [IT. The general result was shown by AIRT as a consequence of their study of preprojective algebras. In this note, we will give a more elementary proof, essentially following the argument of [IT, but without restricting to the Dynkin case.

This note is based on an expository talk given at the Maurice Auslander international conference in 2013. I am very grateful to the organizers for having been invited to speak there, and for their patience in waiting for me to write up the talk. I am also happy to acknowledge financial support from NSERC and the Canada Research Chairs program. I thank the referees for their helpful comments which improved the paper.

\section{Getting started}

Let $Q$ be a quiver without oriented cycles. (It would be possible to work over a general hereditary Artin algebra, but for concreteness, I prefer not to. For parts of this note, e.g. in Section 2, one could also relax the prohibition on oriented cycles in $Q$.) Suppose that $Q$ has $n$ vertices, numbered 1 to $n$.

We write $\operatorname{rep} Q$ for the representations of $Q$ over a fixed ground field $k$. Of our main references, note that DDPW] works over an arbitrary ground field, while Kir works only over an algebraically closed ground field. Since for the topics we present, 
the choice of a general ground field does not introduce any additional complications, we do not assume that $k$ is algebraically closed.

Write $S_{i}$ for the simple representation which has a copy of $k$ at vertex $i$ and the 0 vector space elsewhere.

For $V \in \operatorname{rep} Q$, write $V_{i}$ for the vector space over vertex $i$. Write $\underline{\operatorname{dim}} V$ for the $n$-tuple $\left(\operatorname{dim} V_{1}, \ldots, \operatorname{dim} V_{n}\right) \in \mathbb{Z}^{n}$.

1.1. The Euler-Ringel form. There is an extremely useful bilinear form on $\mathbb{Z}^{n}$.

Theorem 1.1 ([Kir, Theorem 1.25], [DDPW, Proposition 1.9(2)]). The following equation defines a bilinear form on $\mathbb{Z}^{n}$, known as the Euler-Ringel form:

$$
\langle\underline{\operatorname{dim}} V, \underline{\operatorname{dim}} W\rangle=\operatorname{dim} \operatorname{Hom}(V, W)-\operatorname{dim} \operatorname{Ext}^{1}(V, W)
$$

It is not obvious that this defines a bilinear form! In order for it to do so, the righthand side must depend only on $\underline{\operatorname{dim}} V$ and $\underline{\operatorname{dim}} W$, and not on the specific representations $V$ and $W$. On the face of it, one would not expect this to be true. Indeed, the dimensions of $\operatorname{Hom}(V, W)$ and of $\operatorname{Ext}^{1}(V, W)$ do depend on the specific representations, rather than on their dimension vectors. However, taking the difference, we get a quantity which only depends on $\underline{\operatorname{dim}} V$ and $\underline{\operatorname{dim}} W$.

Once we know that this the righthand side only depends on $\underline{\operatorname{dim}} V, \underline{\operatorname{dim}} W$, it is easy to see that it is linear, and to write down an explicit formula for the pairing, because we can choose the most convenient representations of the given dimension vectors. If we want to evaluate $\langle\beta, \gamma\rangle$, the simplest choice is to take semisimple representations, i.e., representations which are direct sums of simple representations. Since $\operatorname{Hom}\left(S_{i}, S_{j}\right)$ is one-dimensional if $i=j$ and zero-dimensional otherwise, and the dimension of $\operatorname{Ext}^{1}\left(S_{i}, S_{j}\right)$ is the number of arrows from $i$ to $j$, we find that

$$
\langle\beta, \gamma\rangle=\sum_{i=1}^{n} \beta_{i} \gamma_{i}-\sum_{(a: i \rightarrow j) \in Q} \beta_{i} \gamma_{j} .
$$

Note that this bilinear form is not symmetric. We should view the fact that we are naturally given a non-symmetric bilinear form as a piece of very good luck. Sometimes, though, it is useful to have a symmetric bilinear form. When we want one, it is easy to get one:

$$
(\beta, \gamma)=\langle\beta, \gamma\rangle+\langle\gamma, \beta\rangle
$$

If, on the contrary, we only had the symmetric form, we would not know how to unsymmetrize it in a meaningful way. (This is not a hypothetical situation! I got into representation theory from Coxeter groups, and, working on Coxeter groups, I became convinced that I needed to break the symmetry of the symmetric bilinear form, but, not knowing anything about quiver representations, I had no idea how to do it.)

1.2. The Weyl group associated to $Q$. For $\beta \in \mathbb{Z}^{n}$ with $(\beta, \beta) \neq 0$, we can define a reflection on $\mathbb{Q}^{n}$, which we denote $t_{\beta}$, by:

$$
t_{\beta}(\gamma)=\gamma-2 \frac{(\beta, \gamma)}{(\beta, \beta)} \beta
$$

This preserves the symmetric form, fixes all vectors orthogonal to $\beta$, and sends $\beta$ to $-\beta$. If $|(\beta, \beta)|$ divides 2 , then $t_{\beta}$ restricts to a map from $\mathbb{Z}^{n}$ to $\mathbb{Z}^{n}$.

It is convenient to let $e_{i}$ denote the standard basis vector consisting of all zeros, with a 1 in the $i$-th position. Equivalently, we can say $e_{i}=\underline{\operatorname{dim}} S_{i}$. 
Let us define $s_{i}=t_{e_{i}}$. Note that, by our definition of the symmetric form, $\left(e_{i}, e_{i}\right)=2$, so $s_{i}=t_{e_{i}}$ is well-defined as a map from $\mathbb{Z}^{n}$ to $\mathbb{Z}^{n}$. We refer to the elements $s_{i}$ as simple reflections.

Define $W$ to be the group generated by the elements $s_{i}$. Since this is a group generated by reflections, it is what is called a reflection group. The generators satisfy some relations which it is easy to verify:

- $s_{i}^{2}=e$,

- if $i$ and $j$ are not adjacent, then $s_{i} s_{j}=s_{j} s_{i}$,

- if there is exactly one arrow between $i$ and $j$, then $s_{i} s_{j} s_{i}=s_{j} s_{i} s_{j}$,

- for clarity, let me add that if there is more than one arrow between $i$ and $j$, then there is no corresponding relation.

It is natural to wonder if there are any other relations. It turns out that there are not.

Theorem 1.2 ([DDPW, Theorem 4.2(1)], Kir, Theorem A.11]). The group $W$ can be defined abstractly as the group generated by $n$ elements $s_{1}, \ldots, s_{n}$ subject to the above relations.

The group $W$ is therefore a Coxeter group. For background on Coxeter groups, see $[\mathrm{BB}, \mathrm{Hu}$.

Let us remark at this point that the group $W$ does not depend on the orientation of the arrows of the quiver; that is to say, it depends only on the underlying graph structure. This is in marked contrast to $\operatorname{rep} Q$, which depends highly on the orientation. Nonetheless, as we shall see, there are connections between the representations of different orientations of the same quiver.

Example 1.1. An example to bear in mind always is the case that the quiver is a path with the vertices numbered 1 to $n$ in order along it, and the arrows oriented arbitrarily. We refer to such a quiver as being of type $A_{n}$. The corresponding Weyl group is isomorphic to the symmetric group on $n+1$ letters; we can take the isomorphism to send $s_{i}$ to the adjacent transposition $(i i+1)$. In examples, we represent an element $w$ of the symmetric group in one-line notation, by writing down the sequence $w(1), \ldots, w(n+1)$.

By definition, each element of $W$ can be expressed as a product of the simple reflections. Such an expression for $w$ of minimal length is called reduced. We write $\ell(w)$ for the length of a reduced expression for $w$. Typically an element $w$ will have many reduced expressions.

For $w \in W$ and $s \in S$, if $w$ has a reduced expression beginning with $s$, then it is easy to see that $\ell(s w)=\ell(w)-1$. It turns out that if $w$ does not have a reduced expression beginning with $s$, then $\ell(s w)=\ell(w)+1$. (When I write "beginning with," I mean with respect to the usual left-to-right order in which English is read.)

Define $\Phi_{r e}=\left\{w e_{i} \mid w \in W, 1 \leq i \leq n\right\}$, and $\Phi_{r e}^{+}=\Phi_{r e} \cap \mathbb{Z}_{\geq 0}^{n}$. The elements of $\Phi_{r e}^{+}$are called the real roots for $Q$.

Example 1.2. For type $A_{n}$, we find that $\Phi_{r e}^{+}$consists of the sums $e_{i}+e_{i+1}+\cdots+e_{j}$ for $1 \leq i \leq j \leq n$.

Let $s_{i_{1}} \ldots s_{i_{r}}$ be a reduced expression for $w \in W$. Define the inversion set corresponding to the reduced expression to be the following set of real roots: 
$\left\{e_{i_{1}}, s_{i_{1}} e_{i_{2}}, s_{i_{1}} s_{i_{2}} e_{i_{3}}, \ldots, s_{i_{1}} s_{i_{2}} \ldots s_{i_{r-1}} e_{i_{r}}\right\}$. This can also be viewed as the collection of positive roots which are sent to negative roots by $w^{-1}$. Note that this latter description does not depend on the choice of reduced expression for $w$; indeed, we have the following proposition:

Proposition 1.1 ([ $\mathrm{BB}$, Corollary 1.4.4, Corollary 1.4.5, and Corollary 3.1.4]). For $w \in W$, all the reduced words for $w$ yield the same inversion set. Different elements of $W$ have different inversion sets. The size of the inversion set of $w$ is $\ell(w)$.

We write $\operatorname{inv}(w)$ for the inversion set of $w$.

Example 1.3. In type $A_{n}$, for $j \geq i$, we have $e_{i}+e_{i+1}+\cdots+e_{j} \in \operatorname{inv}(w)$ if and only if $j+1$ precedes $i$ in the one-line notation for $w$.

In type $A_{2}$, the six elements of $W$, their expressions in one-line notation, and their corresponding inversion sets, are as follows:

$\begin{array}{ccc}e & 123 & \emptyset \\ s_{1} & 213 & \left\{e_{1}\right\} \\ s_{2} & 132 & \left\{e_{2}\right\} \\ s_{1} s_{2} & 231 & \left\{e_{1}, e_{1}+e_{2}\right\} \\ s_{2} s_{1} & 312 & \left\{e_{2}, e_{1}+e_{2}\right\} \\ s_{1} s_{2} s_{1} & 321 & \left\{e_{1}, e_{1}+e_{2}, e_{2}\right\}\end{array}$

We have listed the inversion sets in the order corresponding to the reduced expressions given above. If we take a different reduced word for the same element, considering $s_{2} s_{1} s_{2}$ instead of $s_{1} s_{2} s_{1}$, we get the roots $e_{2}, e_{1}+e_{2}, e_{1}$, in that order. Consistent with Proposition 1.1, the set of roots is the same as appears in the table for $s_{1} s_{2} s_{1}$, though the order in which they appear is different.

1.3. Finite versus infinite. There is a basic dichotomy in root system combinatorics. In our setting, it manifests in the following theorem:

Theorem 1.3. The following are equivalent:

- $W$ is finite,

- $\operatorname{rep} Q$ has only finitely many indecomposables up to isomorphism (in which case we say $Q$ is of finite representation type),

- $Q$ consists of a finite number of Dynkin quivers, i.e., quivers of type $A_{n}$ $(n \geq 1), D_{n}(n \geq 4), E_{6}, E_{7}$, or $E_{8}$.

We have already met quivers of type $A_{n}$. A quiver of type $D_{n}$ is a quiver with $n$ vertices, obtained by orienting the following graph:

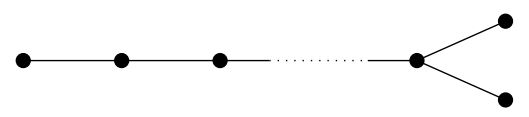

The quivers of type $E_{6}, E_{7}$, and $E_{8}$ are obtained by orienting the graphs shown below:
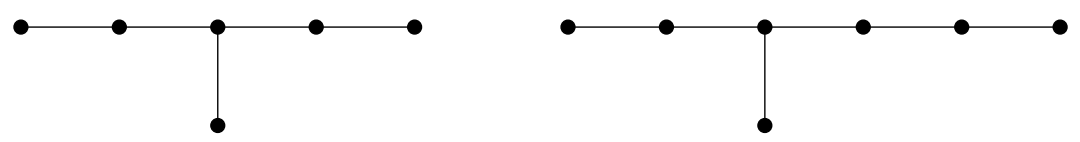


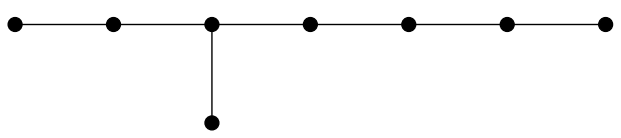

The equivalence of (ii) and (iii) is the celebrated theorem of Gabriel DDPW, Theorem 1.23], Kir, Theorem 3.3]. The equivalence of (i) and (iii) follow from the classification of finite Coxeter groups, see for example [Kir, Theorem A.11].

\section{REAL AND IMAGINARY ROOTS}

It turns out that $\Phi_{r e}^{+}$, as defined above, is intimately connected to the representation theory of $Q$.

Theorem 2.1 ([Kir, Theorem 7.49], DDPW, Remark 1.26]). There is a unique indecomposable representation of $Q$ whose dimension vector is $\alpha$ for each $\alpha \in \Phi_{r e}^{+}$.

This theorem was established under the assumption that $k$ is algebraically closed by Kac Kac. As pointed out by Schofield Sch], Kac's proof also applies to any field of characteristic $p$. The characteristic zero case is proved in Sch. Note that exceptionally [DDPW] only states the result for algebraically closed ground fields.

If $Q$ is of finite representation type, then the representations of Theorem 2.1 exhaust the indecomposables. However, if $Q$ is not of finite type, there is another class of roots, the imaginary roots.

Let $M$ be the cone in $\mathbb{Z}_{>0}^{n}$ consisting of vectors with connected support and such that for $\alpha \in M$, we have $\left(\alpha, e_{i}\right) \leq 0$. The imaginary roots are all elements of $\mathbb{Z}^{n}$ of the form $w \alpha$ for $w \in W$ and $\alpha \in M$. The following result is due to Kac $\mathrm{Kac}$ :

Theorem 2.2 ([ $\mathrm{Kir}$, Theorem 7.49 and Theorem A.20]). Let $k$ be algebraically closed. The dimension vectors of indecomposable representations of $Q$ are the positive real roots together with the imaginary roots. The positive real roots are those dimension vectors for which there exists a unique indecomposable representation, while the imaginary roots are those dimension vectors for which there are infinitely many non-isomorphic indecomposable representations.

This theorem obviously requires some modification to accommodate finite fields; in fact, a full analogue of this theorem for general fields is not known.

\section{REFLECTION FUnCTORS}

In this section we define the reflections functors, which play a decisive role in the representation theory of quivers of finite representation type, but which, as we will see, can also be important for general quiver representations. The recognition of their importance goes back to the classic paper of Bernstein-Gelfand-Ponomarev BGP.

We say that a vertex $i$ is a sink of $Q$ if all the arrows incident to $i$ point towards $i$. Dually, we say $i$ is a source if all arrows incident to $i$ point away from it.

If $i$ is a sink or a source, we define $\mu_{i}(Q)$ to be the quiver obtained by changing the direction of all the arrows incident to $i$.

Suppose that $i$ is a sink of $Q$. Let $V$ be a representation of $Q$. We want to define a representation $R_{i}^{+}(V)$ of $Q^{\prime}=\mu_{i}(Q)$.

First, we define the vector spaces: 


$$
R_{i}^{+}(V)_{j}= \begin{cases}V_{j} & \text { for } j \neq i \\ \operatorname{ker}\left(\bigoplus_{(a: j \rightarrow i) \in Q} V_{j}\right) \rightarrow V_{i} & \text { for } j=i\end{cases}
$$

Note that the direct sum runs over all arrows pointing to $i$ in $Q$. In the case that there is more than one arrow from $j$ to $i$, the summand $V_{j}$ will be repeated.

We also have to define the linear maps associated to the arrows of $\mu_{i}(Q)$. For the arrows which are also in $Q$, we notice that neither the source nor the target vector spaces of the arrow have changed, so we can and do simply reuse the map from $V$. It remains to define the maps corresponding to the arrows in $\mu_{i}(Q)$ which point away from $i$. We use the maps coming from the natural inclusion of $R_{i}^{+}(V)_{i}$ into the sum over all the arrows from $j$ to $i$ in $Q$ of $V_{j}$.

Example 3.1. Let $Q$ be the quiver of type $A_{3}$ shown below. Then $Q^{\prime}=\mu_{2}(Q)$ is also as shown.
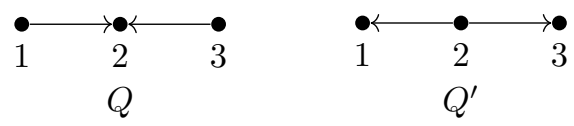

Below, in the lefthand column, we list the six indecomposable representations of $Q$, and in the righthand column, the representation of $Q^{\prime}$ to which it is sent by $R_{2}^{+}$ (which is indecomposable or, in the first case, the zero representation). We only give the vector spaces at each vertex; the maps between consecutive copies of $k$ are non-zero.
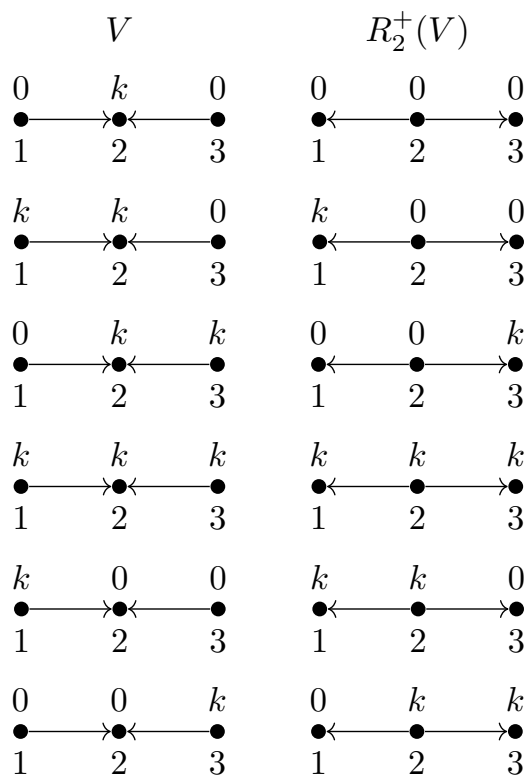

In order for $R_{i}^{+}$to be a functor, for $f \in \operatorname{Hom}(V, W)$, we must define $R_{i}^{+}(f) \in$ $\operatorname{Hom}\left(R_{i}^{+}(V), R_{i}^{+}(W)\right)$. For $j \neq i$, we define $R_{i}^{+}(f)_{j}=f_{j}$. To define $R_{i}^{+}(f)_{i}$, we consider the following commutative diagram: 


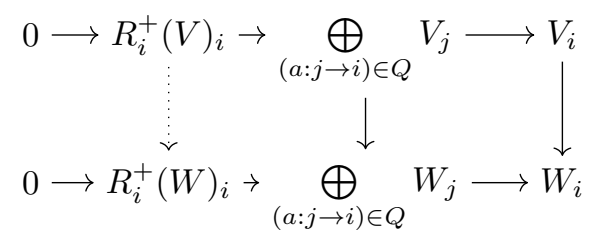

The rows of the diagram are the exact sequences which define $R_{i}^{+}(V)_{i}$ and $R_{i}^{+}(W)_{i}$. The vertical undotted arrows are maps defined by $f$; the dotted arrow is the arrow we want to define. We can see in the diagram a map from $R_{i}^{+}(V)_{i}$ to $\bigoplus_{(a: j \rightarrow i)} W_{j}$. We observe that if we compose it with the map from $\bigoplus_{(a: j \rightarrow i)} W_{j}$ to $W_{i}$, the result will be zero, which means that in fact the image of $R_{i}^{+}(V)_{i}$ falls in $R_{i}^{+}(W)_{i}$. We take this map from $R_{i}^{+}(V)_{i}$ to $R_{i}^{+}(W)_{i}$ to be the definition of $R_{i}^{+}(f)_{i}$.

We have gone into considerable detail describing $R_{i}^{+}$; the definition of $R_{i}^{-}$which is defined when $i$ is a source is dual in the natural way. Let me mention only the key point that $R_{i}^{-}(V)_{i}$ is defined to be the cokernel of the natural map from $V_{i}$ to $\bigoplus_{(a: i \rightarrow j)} V_{j}$. We then have the following theorem:

Theorem 3.1 ([DDPW, Theorem 1.18], [Kir, Theorem 3.10]). Let $i$ be a sink in $Q$, and let $Q^{\prime}=\mu_{i}(Q)$. Then $R_{i}^{+}$is a functor from representations of $Q$ to representations of $Q^{\prime}$, and $R_{i}^{-}$is a functor from representations of $Q^{\prime}$ to representations of $Q$.

Let us note some important properties of $R_{i}^{+}$and $R_{i}^{-}$.

Proposition 3.1. Let $i$ be a sink in $Q$, and let $Q^{\prime}=\mu_{i}(Q)$. We write $S_{i}$ for the simple representation at $i$ of $Q$, and $S_{i}^{\prime}$ for the simple representation at $i$ of $Q^{\prime}$.

(1) $R_{i}^{+}\left(S_{i}\right)=0$ and $R_{i}^{-}\left(S_{i}^{\prime}\right)=0$.

(2) If $V \in \operatorname{rep} Q$ has no direct summand isomorphic to $S_{i}$, then

$$
\underline{\operatorname{dim}} R_{i}^{+}(V)=s_{i}(\underline{\operatorname{dim}}(V)) .
$$

Similarly if $V^{\prime} \in \operatorname{rep} Q^{\prime}$ has no direct summand isomorphic to $S_{i}^{\prime}$ then

$$
\underline{\operatorname{dim}} R_{i}^{-}\left(V^{\prime}\right)=s_{i}\left(\underline{\operatorname{dim}}\left(V^{\prime}\right)\right) \text {. }
$$

(3) $R_{i}^{+}$is left exact; $R_{i}^{-}$is right exact.

(4) If $V \in \operatorname{rep} Q$, then $R_{i}^{-}\left(R_{i}^{+}(V)\right)$ is isomorphic to the direct sum of the indecomposable summands of $V$ which are not isomorphic to $S_{i}$ (and a similar dual statement).

(5) The image of $R_{i}^{+}(\operatorname{rep} Q)$ consists of all representations of $Q^{\prime}$ not having $S_{i}^{\prime}$ as an indecomposable summand (and a similar dual statement).

Proof. (1) is immediate from the definition.

For (2), we prove on the first statement (about $R_{i}^{+}$). Suppose that $V$ has no direct summands isomorphic to $S_{i}$. In this case, the map $\bigoplus_{(a: j \rightarrow i)} V_{j} \rightarrow V_{i}$ is surjective. This means that

$$
\operatorname{dim} R_{i}^{+}(V)_{i}=\left(\sum_{(a: j \rightarrow i) \in Q} \operatorname{dim} V_{j}\right)-\operatorname{dim} V_{i}
$$


while $\operatorname{dim} R_{i}^{+}(V)_{j}=\operatorname{dim} V_{j}$ for $j \neq i$. This claim follows. Property (2) explains both the name ("reflection functor") and one of the reasons that this topic is included in this note.

For (3), the statement is clear except over vertex $i$. There, it follows from an application of the Snake Lemma. For another proof, see [Kir, Theorem 3.10] or DDPW, Theorem 1.18].

(4) follows from applying (2) to the decomposition of $V$ into indecomposables.

For (5), we check directly that for $M \in \operatorname{rep} Q$, we have that $R_{i}^{+}(M)$ has no $S_{i}^{\prime}$ direct summand. The fact that all representations without such a summand are in the image follows from (4).

At the beginning of this section, we defined $\mu_{i}(Q)$ to be the result of reversing the arrows incident to $i$, provided that $i$ was either a sink or a source. Clearly, this definition could still be applied if $i$ is neither a sink nor a source, but it turns out that, from the point of view of representation theory, it is not the right thing to do: the right thing is to apply (Fomin-Zelevinsky) mutation, but the whole story becomes more complicated. See [DWZ] for more on this.

\section{TORSION FREE ClASSES AND $c$-SORTABLE ELEMENTS}

We are now ready to introduce $c$-sortable elements of $W$ and finite torsion free classes in $\operatorname{rep} Q$. We will see that they have very similar inductive structures, which we will exploit to construct a bijection between them.

4.1. The correspondence between Coxeter elements and orientations of a quiver. Let $|Q|$ be the unoriented graph underlying the quiver $Q$. As was already mentioned, the Weyl group associated to $Q$ only depends on $|Q|$. A Coxeter element for $W$ is, by definition, a product of each of the Coxeter generators of $W$ in some order. The following fact is easy but important.

Lemma 4.1. There is a bijection between the acyclic orientations of $|Q|$ and the Coxeter elements of $W$.

Proof. Associate to $Q$ the product of the Coxeter generators in which $s_{i}$ precedes $s_{j}$ if there are one or more arrows from $j$ to $i$ in the quiver. This does not prescribe the order of all pairs of Coxeter generators, but any pair whose order is not prescribed is a pair whose corresponding vertices are not connected by an arrow, and which therefore commute. The fact that it is possible to find a linear order on the generators satisfying this prescription follows from the fact that the orientation of $Q$ has no cycles.

Conversely, any Coxeter element determines an acyclic orientation of $|Q|$ by orienting any edges between $i$ and $j$ towards $i$ if $s_{i}$ precedes $s_{j}$ in the Coxeter element.

We write $c_{Q}$ for the Coxeter element associated to $Q$.

Example 4.1. Let $Q_{1}$ and $Q_{2}$ be as shown below:
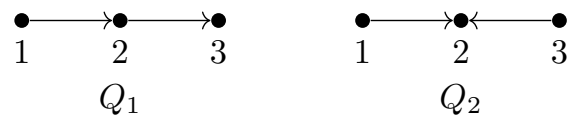
In the Coxeter element associated to $Q_{1}$, we have $s_{3}$ before $s_{2}$ and $s_{2}$ before $s_{1}$, so $c_{Q_{1}}=s_{3} s_{2} s_{1}$. In the Coxeter element associated to $Q_{2}$, we have $s_{2}$ before $s_{1}$ and $s_{2}$ before $s_{3}$. We therefore could write $c_{Q_{2}}=s_{2} s_{1} s_{3}$ or $c_{Q_{2}}=s_{2} s_{3} s_{1}$; these are equal because $s_{1} s_{3}=s_{3} s_{1}$.

4.2. Definition of $c$-sortable elements. Let $c=s_{i_{1}} \ldots s_{i_{n}}$ be a Coxeter element for $W$. For $J \subseteq\{1, \ldots, n\}$, we write $c_{J}$ for the subword of $c$ whose reflections are those $s_{j}$ with $j \in J$.

An element $w \in W$ is called $c$-sortable if it has a reduced expression $w=$ $c_{J_{1}} c_{J_{2}} \ldots c_{J_{k}}$ with $J_{1} \supseteq J_{2} \supseteq \cdots \supseteq J_{k}$.

Example 4.2. In the Weyl group of type $A_{2}$, isomorphic to $S_{3}$, setting $c=s_{1} s_{2}$, five of the six group elements are $c$-sortable. The one which is not is $s_{2} s_{1}$. In the Weyl group of type $A_{3}$, for each choice of $c$ there are $14 c$-sortable elements. In general, the $c$-sortable elements in type $A_{n}$ are counted by the $n$-th Catalan number, $\frac{1}{n+2}\left(\begin{array}{c}2 n+2 \\ n+1\end{array}\right)$. See [Re, Theorem 9.1], which gives complete information about enumeration in all finite types.

The $c$-sortable elements of a Coxeter group $W$ were defined by Reading $[\mathrm{Re}$ as a bridge between the combinatorics of finite-type cluster algebras and the noncrossing partitions of $W$. They play a central role in the Coxeter-theoretic approach to the combinatorics of cluster algebras, as developed by Reading and Speyer in a sequence of articles [RS1, RS2, RS3; they have also turned out to have interesting applications in the representation theory of preprojective algebras, see [AIRT.

There is an extremely useful inductive characterization of $c$-sortable elements. In the theorem below, we use the notation $W_{\left\langle s_{j}\right\rangle}$ for the Coxeter group generated by the reflections $s_{i}$ for $i \neq j$. If $W$ is the Coxeter group corresponding to the quiver $Q$, then $W_{\left\langle s_{j}\right\rangle}$ is the Coxeter group for the quiver with vertex $j$ removed. (While initially counter-intuitive, this notation is standard and very useful.)

Theorem 4.1 ([Re, Lemma 2.4, Lemma 2.5, and Remark 2.7]). Let $w \in W$, and let $c=s_{i_{1}} \ldots s_{i_{n}}$.

(1) If $\ell\left(s_{i_{1}} w\right)>\ell(w)$, then $w$ is c-sortable iff $w$ is a $s_{i_{2}} \ldots s_{i_{n}}$-sortable element in $W_{\left\langle s_{i_{1}}\right\rangle}$.

(2) If $\ell\left(s_{i_{1}} w\right)<\ell(w)$, then $w$ is c-sortable iff $s_{i_{1}} w$ is $s_{i_{2}} s_{i_{3}} \ldots s_{i_{n}} s_{i_{1}}$-sortable.

Proof. Suppose first that $\ell\left(s_{i_{1}} w\right)>\ell(w)$. This means that no reduced expression for $w$ can begin with $s_{i_{1}}$. If $w$ is $c$-sortable, then, since it does not use the $s_{i_{1}}$ in the first copy of $c$, it can never use any $s_{i_{1}}$. Therefore it is in $W_{\left\langle s_{i_{1}}\right\rangle} ;$ the $s_{i_{2}} \ldots s_{i_{n}}$ sortability there is immediate; the converse is also clear.

Now suppose that $\ell\left(s_{i_{1}} w\right)<\ell(w)$. This means that there is a reduced expression for $w$ beginning with $s_{i_{1}}$. Suppose that $w$ is $c$-sortable. Since $w \notin W_{\left\langle s_{\left.i_{1}\right\rangle}\right\rangle}$, any expression for $w$ must use $s_{i_{1}}$ at least once, so the expression which manifests that $w$ is $c$-sortable must begin with $s_{i_{1}}$. Therefore, it expresses $w$ as $s_{i_{1}}$ times a reduced word for $s_{i_{1}} w$, which, when we unwind the definition, must be $s_{i_{2}} \ldots s_{i_{n}} s_{i_{1}}$-sortable. This is exactly what we wanted. The converse argument works the same way.

Example 4.3. In type $A_{2}$, with $c=s_{1} s_{2}$, the first case of Theorem 4.1 applies to the $c$-sortable elements $e$ and $s_{2}$, while the second case applies to $s_{1}, s_{1} s_{2}$, and $s_{1} s_{2} s_{1}$, The non-c-sortable element $s_{2} s_{1}$ falls into the first case of the theorem, which says that it is not $c$-sortable because it is not an $s_{2}$-sortable element of $W_{\left\langle s_{1}\right\rangle}$ (obviously true, since it is not an element of $W_{\left\langle s_{1}\right\rangle}$ at all). 
4.3. The correspondence between $c$-sortable elements and finite torsion free classes. A torsion free class in $\operatorname{rep} Q$ is a full additive subcategory closed under extensions and subrepresentations. That is to say, if $\mathcal{F}$ is a torsion free class and $Y$ is in $\mathcal{F}$, so are all the subrepresentations of $Y$, and if $X, Z \in \mathcal{F}$, such that there is an extension

$$
0 \rightarrow X \rightarrow Y \rightarrow Z \rightarrow 0
$$

then $Y \in \mathcal{F}$.

We say that a torsion free class is finite if it contains finitely many indecomposable representations up to isomorphism. If $Q$ is a Dynkin quiver, then all torsion free classes will be finite. Conversely, if all torsion free classes in $\operatorname{rep} Q$ are finite, then $Q$ is a Dynkin quiver, since the category of all representations is itself a torsion free class.

For $w$ a $c_{Q}$-sortable element of $W$, define $\mathcal{F}(w)$ to be the full subcategory of rep $Q$ consisting of direct sums of copies of the indecomposable representations whose dimension vectors are roots $\operatorname{in} \operatorname{inv}(w)$.

We can now state the theorem whose proof occupies the remainder of the paper.

Theorem 4.2. The map $\mathcal{F}$ is a bijection from $c_{Q}$-sortable elements of $W$ to finite torsion free classes in $\operatorname{rep} Q$.

Example 4.4. Let $Q$ be the quiver $1 \leftarrow 2$. Then the correspondence between $c_{Q}$-sortable elements of $W$ and torsion free classes is as follows:

$\begin{array}{cc}w & \mathcal{F}(w) \\ e & 0 \\ s_{1} & \operatorname{add} S_{1} \\ s_{2} & \operatorname{add} S_{2} \\ s_{1} s_{2} & \operatorname{add} S_{1}, P_{2} \\ s_{1} s_{2} s_{1} & \operatorname{add} S_{1}, P_{2}, S_{2}\end{array}$

Here, we have written $P_{2}$ for the non-simple indecomposable representation, and we have written add for the additive category generated by the subsequent list of indecomposable representations. As is easly verified, the righthand column consists of the torsion free classes of $\operatorname{rep} Q$.

4.4. The inductive structure of torsion free classes. In order to prove Theorem 4.2, we will exhibit an inductive structure to torsion free classes which parallels the inductive structure of $c$-sortable elements. Suppose $\mathcal{F}$ is a torsion free class for rep $Q$. Let $i$ be a sink of $Q$, so $S_{i}$ is a simple projective. We want to prove a structure theorem about $\mathcal{F}$, parallel to Theorem 4.1. We split into two cases, based on whether $S_{i} \in \mathcal{F}$.

Proposition 4.1. If $S_{i} \notin \mathcal{F}$, then $\mathcal{F}$ is supported on $Q \backslash\{i\}$, and thus defines a torsion free class in the representations of $Q \backslash\{i\}$.

Proof. Recall that for $M \in \operatorname{rep} Q$, we have that $\operatorname{dim} \operatorname{Hom}\left(S_{i}, M\right)=\operatorname{dim} M_{i}$, since $S_{i}$ is projective. Thus, if $M_{i} \neq 0$, then $\operatorname{Hom}\left(S_{i}, M\right) \neq 0$. And note that in this case, the nonzero maps from $S_{i}$ to $M$ would have to be injections, because $S_{i}$ has no non-zero subrepresentations. That means that if $M_{i} \neq 0$, we must have that $M \notin \mathcal{F}$, since otherwise the inclusion of $S_{i}$ into $M$ would contradict the fact that $S_{i} \notin \mathcal{F}$ but $M \in \mathcal{F}$. 
Proposition 4.2. If $S_{i} \in \mathcal{F}$, then $R_{i}^{+} \mathcal{F}$ is a torsion free class for $\mu_{i}(Q)$ which does not contain $S_{i}$. If $\mathcal{F}$ contains finitely many indecomposable representations up to isomorphism, then $\mathcal{F}^{\prime}$ contains one fewer.

Proof. Let us write $Q^{\prime}$ for $\mu_{i}(Q)$. We will write $X^{\prime}, Y^{\prime}$, etc. for representations of $Q^{\prime}$ and $X, Y$, etc. for representations of $Q$. Let $\mathcal{F}^{\prime}=R_{i}^{+} \mathcal{F}$. We begin by proving that $\mathcal{F}^{\prime}$ is closed under subrepresentations. Let $X^{\prime} \in \mathcal{F}^{\prime}$. Since $X^{\prime} \in$ $R_{i}^{+}(\operatorname{rep} Q)$, we know that $X^{\prime}$ has no direct summands isomorphic to $S_{i}^{\prime}$. Let $Y^{\prime}$ be a subrepresentation of $X^{\prime}$.

Let $Y=R_{i}^{-}\left(Y^{\prime}\right)$ and $X=R_{i}^{-}\left(X^{\prime}\right)$. Since $X^{\prime} \in \mathcal{F}^{\prime}$, we know that $X \in \mathcal{F}$. Since $R_{i}^{-}$is only right exact, it is not necessarily the case that $Y$ is a subrepresentation of $X$. However, $R_{i}^{-}$applied to the inclusion from $Y^{\prime}$ to $X^{\prime}$ gives a map from $Y$ to $X$. The image of this map is a subrepresentation of $X$, so lies in $\mathcal{F}$, and the kernel of this map is a sum of copies of $S_{i}$, which also lies in $\mathcal{F}$. Thus, since $\mathcal{F}$ is extension closed, it also contains $Y$, and thus $Y^{\prime} \in \mathcal{F}^{\prime}$, showing that $\mathcal{F}^{\prime}$ is closed under subrepresentations.

We next check that $\mathcal{F}^{\prime}$ is closed under extensions. Suppose that we have an extension of $Q^{\prime}$ representations:

$$
0 \rightarrow Y^{\prime} \rightarrow X^{\prime} \rightarrow Z^{\prime} \rightarrow 0
$$

with $Y^{\prime}$ and $Z^{\prime}$ in $\mathcal{F}^{\prime}$.

Any indecomposable representation of $Q^{\prime}$ that admits a nonzero morphism from $S_{i}^{\prime}$ is isomorphic to $S_{i}^{\prime}$. By assumption, neither $Y^{\prime}$ nor $Z^{\prime}$ has an $S_{i}^{\prime}$ direct summand, so $\operatorname{Hom}\left(S_{i}^{\prime}, Y^{\prime}\right)=0=\operatorname{Hom}\left(S_{i}^{\prime}, Z^{\prime}\right)$. By the Hom long exact sequence, it follows that $\operatorname{Hom}\left(S_{i}^{\prime}, X^{\prime}\right)=0$, so $X^{\prime}$ does not have any direct summands isomorphic to $S_{i}^{\prime}$ either. Applying $R_{i}^{-}$, we get

$$
R_{i}^{-}\left(Y^{\prime}\right) \rightarrow R_{i}^{-}\left(X^{\prime}\right) \rightarrow R_{i}^{-}\left(Z^{\prime}\right) \rightarrow 0,
$$

since $R_{i}^{-}$is right exact. Since none of $X^{\prime}, Y^{\prime}, Z^{\prime}$ have any $S_{i}^{\prime}$ summands, the dimension vector of $R_{i}^{-}\left(X^{\prime}\right)$ is $s_{i}\left(\underline{\operatorname{dim}}\left(X^{\prime}\right)\right)$, and similarly for $Y^{\prime}$ and $Z^{\prime}$. Since $s_{i}$ is a linear map, $s_{i} \underline{\operatorname{dim}}\left(X^{\prime}\right)=s_{i} \underline{\operatorname{dim}}\left(Y^{\prime}\right)+s_{i} \underline{\operatorname{dim}}\left(Z^{\prime}\right)$, and it follows that (10) is exact on the left as well. Now $R_{i}^{-}(Y)$ and $R_{i}^{-}(Z)$ are in $\mathcal{F}$, so, since (11) is exact on the left as well, we can conclude that $R_{i}^{-}\left(X^{\prime}\right)$ is also in $\mathcal{F}$, and $X^{\prime} \in \mathcal{F}^{\prime}$, as desired.

The final statement follows from the fact that $R_{i}^{+}$sends non-isomorphic indecompolsables other than $S_{i}$ to non-isomorphic indecomposables, while killing $S_{i}$.

The previous two propositions tell us that, given $i$ a sink of $Q$ and $\mathcal{F}$ a torsion free class in $\operatorname{rep} Q$, there is a way to associate to it a torsion free class that is "smaller" in a suitable sense: either smaller because it is defined on a smaller quiver (the case of Proposition 4.1) or smaller because it contains fewer indecomposables (the case of Proposition 4.2.

We now want to reverse the process and show that, given a candidate "smaller" torsion free class, it is possible to recover a torsion free class in $\operatorname{rep} Q$.

The first case, reversing Proposition 4.1 is obvious, but we state it to be able to refer to it later.

Proposition 4.3. If $\mathcal{F}$ is a torsion free class in the category of representations of $Q \backslash\{i\}$, then it is also a torsion free class in the category of representations of $Q$, via the inclusion of $\operatorname{rep} Q \backslash\{i\}$ into $\operatorname{rep} Q$. 
The second case, reversing Proposition 4.2 requires a proof, but it is similar to that of Proposition 4.2 .

Proposition 4.4. Let $\mathcal{F}^{\prime}$ be a torsion free class in $\operatorname{rep} Q^{\prime}$. Let $\mathcal{F}$ be the additive hull of $R_{i}^{-} \mathcal{F}^{\prime}$ and $S_{i}$. Then $\mathcal{F}$ is a torsion free class in $\operatorname{rep} Q$.

Proof. Let $X$ be in $\mathcal{F}$, and let $Y$ be a subrepresentation of $X$. We want to show that $Y \in \mathcal{F}$. Let $X^{\prime}=R_{i}^{+}(X)$, and let $Y^{\prime}=R_{i}^{+}(Y)$. Since $R_{i}^{+}$kills $S_{i}, R_{i}^{+} \mathcal{F}=$ $R_{i}^{+}\left(R_{i}^{-} \mathcal{F}^{\prime}\right) \subseteq \mathcal{F}^{\prime}$. Since $R_{i}^{+}$is left exact, $Y^{\prime}$ is a subrepresentation of $X^{\prime}$, and thus is in $\mathcal{F}^{\prime}$. Now $Y$ is isomorphic to the direct sum of $R_{i}^{+}\left(Y^{\prime}\right)$ with some copies of $S_{i}$, and both of these are in $\mathcal{F}$, so $Y$ is in $\mathcal{F}$.

Now suppose that we have a short exact sequence

$$
0 \rightarrow Y \rightarrow X \rightarrow Z \rightarrow 0
$$

with $Y$ and $Z$ in $\mathcal{F}$. We want to show that $X \in \mathcal{F}$.

Applying $R_{i}^{+}$, we get an exact sequence:

$$
0 \rightarrow R_{i}^{+}(Y) \rightarrow R_{i}^{+}(X) \rightarrow R_{i}^{+}(Z)
$$

By our assumptions, $R_{i}^{+}(Y)$ and $R_{i}^{+}(Z)$ are both in $\mathcal{F}^{\prime}$. In order to get exactness on the right, we can replace $R_{i}^{+}(Z)$ by the cokernel of the map from $R_{i}^{+}(Y)$ to $R_{i}^{+}(X)$. Note that it is isomorphic to a subrepresentation of $R_{i}^{+}(Z)$, and therefore it is also in $\mathcal{F}^{\prime}$. It follows that $R_{i}^{+}(X) \in \mathcal{F}^{\prime}$, and thus that $X$ is in $\mathcal{F}$.

Note that we actually only use the above proposition in the case that $\mathcal{F}^{\prime}$ does not contain $S_{i}^{\prime}$, but we give the more general statement, since the proof is no different.

4.5. Proof of Theorem 4.2, We will prove Theorem 4.2 by establishing the next two propositions.

Proposition 4.5. Let $\mathcal{F}$ be a finite torsion free class in $\operatorname{rep} Q$. Then there is some $c$-sortable element $w$ such that $\mathcal{F}=\mathcal{F}(w)$.

Proof. The proof is by induction on the number of vertices of $Q$ and the number of indecomposable representations of $\mathcal{F}$.

Let the first reflection in $c_{Q}$ be $s_{i}$. By the definition of $c_{Q}$, vertex $i$ is a sink of $Q$. We split into two cases depending on whether or not $S_{i} \in \mathcal{F}$.

If $S_{i} \notin \mathcal{F}$, then by Proposition 4.1 $\mathcal{F}$ is a torsion free class in the representations of $Q \backslash\{i\}$. By induction, there is a $c_{Q \backslash\{i\}}$-sortable element $w$ of $W_{\langle i\rangle}$ such that $\mathcal{F}=\mathcal{F}(w)$. This $w$ is also $c_{Q}$-sortable in $W$ by Theorem 4.1, and we are done.

If $S_{i} \in \mathcal{F}$, then by Proposition 4.2. $\mathcal{F}^{\prime}=R_{i}^{+} \mathcal{F}$ is a torsion free class for $Q^{\prime}=$ $\mu_{i}(Q)$. By induction, $\mathcal{F}^{\prime}=\mathcal{F}\left(w^{\prime}\right)$ for $w^{\prime}$ a $c_{Q^{\prime}}$-sortable element. Since $\mathcal{F}^{\prime}$ does not contain $S_{i}^{\prime}$, we know $\operatorname{inv}\left(w^{\prime}\right)$ does not contain $e_{i}$. Therefore, no reduced expression for $w^{\prime}$ begins with $s_{i}$, and $\ell\left(s_{i} w^{\prime}\right)=\ell\left(w^{\prime}\right)+1$. Let $w=s_{i} w^{\prime}$. Since $c_{Q^{\prime}}=s_{i} c_{Q} s_{i}$, Theorem 4.1 tells us that $w$ is $c_{Q}$-sortable. Now:

$$
\begin{aligned}
\operatorname{inv}(w) & =\left\{e_{i}\right\} \cup\left\{s_{i} \alpha \mid \alpha \in \operatorname{inv}\left(w^{\prime}\right)\right\} \\
& =\left\{\underline{\operatorname{dim}} S_{i}\right\} \cup\left\{s_{i} \underline{\operatorname{dim}} M^{\prime} \mid M^{\prime} \text { indecomposable in } \mathcal{F}^{\prime}\right\} \\
& =\{\underline{\operatorname{dim}} M \mid M \text { indecomposable in } \mathcal{F}\},
\end{aligned}
$$

and this shows that $w$ is the desired $c$-sortable element of $W$.

Proposition 4.6. Let $w$ be a $c_{Q}$-sortable element of $W$. Then $\mathcal{F}(w)$ is a finite torsion free class. 
Proof. The proof is by induction on the number of vertices of $Q$ and the number of inversions of $w$. Let the first reflection of $c_{Q}$ be $s_{i}$. Then vertex $i$ is a sink of $Q$. We split into two cases depending on whether $\ell\left(s_{i} w\right)>\ell(w)$ or $\ell\left(s_{i} w\right)<\ell(w)$.

Suppose first that $\ell\left(s_{i} w\right)>\ell(w)$. Since $w$ is $c_{Q}$-sortable, Theorem 4.1 tells

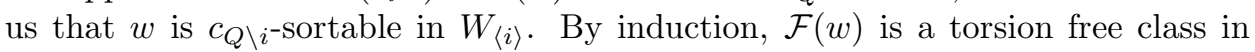
$\operatorname{rep} Q \backslash\{i\}$. Now Proposition 4.3 tells us that $F(w)$ is also a torsion free class in $\operatorname{rep} Q$.

Suppose next that $\ell\left(s_{i} w\right)<\ell(w)$. Theorem 4.1 tells us that $s_{i} w$ is $s_{i} c_{Q} s_{i^{-}}$ sortable. We note that this means that $s_{i} w$ is $c_{\mu_{i}(Q)}$-sortable, so, by induction, $\mathcal{F}\left(s_{i} w\right)$ is a torsion free class for $\mu_{i}(Q)$. We know that

$$
\begin{aligned}
\operatorname{inv}(w) & =\left\{e_{i}\right\} \cup\left\{s_{i} \alpha \mid \alpha \in \operatorname{inv}\left(s_{i} w\right)\right\} \\
& =\left\{\underline{\operatorname{dim}} S_{i}\right\} \cup\left\{\underline{\operatorname{dim}} R_{i}^{-} M \mid M \text { indecomposable in } \mathcal{F}\left(s_{i} w\right)\right\}
\end{aligned}
$$

Proposition 4.4 now tells us that $\mathcal{F}(w)$ is a torsion free class, as desired.

This completes the proof of Theorem 4.2

\section{REFERENCES}

[AIRT] C. Amiot, O. Iyama, I. Reiten, and G. Todorov. Preprojective algebras and $c$-sortable words. Proc. Lond. Math. Soc. (3) 104 (2012), no. 3, 513-539.

[BGP] I. N. Bernšteřn, I. M. Gel'fand, and V. A. Ponomarev. Coxeter functors, and Gabriel's theorem. (Russian) Uspehi Mat. Nauk 28 (1973), no. 2(170), 19-33. English translation: Russian Math. Surveys 28 (1973), no. 2, 17-32.

[BB] A. Björner and F. Brenti. Combinatorics of Coxeter groups. Springer, New York, 2005.

[DDPW] B. Deng, J. Du, B. Parshall, and J. Wang. Finite dimensional algebras and quantum groups. American Mathematical Society, Providence, RI, 2008.

[DWZ] H. Derksen, J. Weyman, and A. Zelevinsky. Quivers with potentials and their representations. I. Mutations. Selecta Math. (N.S.) 14 (2008), no. 1, 59-119.

[Hu] J. Humphreys. Reflection groups and Coxeter groups. Cambridge University Press, Cambridge, 1990.

[IT] C. Ingalls and H. Thomas. Noncrossing partitions and representations of quivers. Compos. Math. 145 (2009), no. 6, 1533-1562.

[Kac] V. Kac. Infinite root systems, representations of graphs and invariant theory. Invent. Math. 56 (1980), no. 1, 57-92.

[Kir] A. Kirillov. Quiver representations and quiver varieties. American Mathematical Society, Providence, RI, 2016.

[Sch] A. Schofield. The field of definition of a real representation of a quiver Q. Proceedings of the American Mathematical Society 116 (1992), no. 2, 293-295.

[Re] N. Reading. Clusters, Coxeter-sortable elements and noncrossing partitions. Trans. Amer. Math. Soc. 359 (2007), no. 12, 5931-5958.

[RS1] N. Reading and D. Speyer. Cambrian fans. J. Eur. Math. Soc. 11 (2009), no. 2, 407-447.

[RS2] N. Reading and D. Speyer. Sortable elements in infinite Coxeter groups. Trans. Amer. Math. Soc. 363 (2011), no. 2, 699-761.

[RS3] N. Reading and D. Speyer. Cambrian frameworks for cluster algebras of finite type. Trans. Amer. Math. Soc 370 (2018), no. 2, 1429-1468.

Département de mathématiques, Université du Québec À Montréal, Montréal, Québec, Canada

E-mail address: hugh.ross.thomas@gmail.com 